\title{
A Fusion Hybrid Propulsion System for Rapid Interplanetary Transport
}

\author{
T. Kammash* \\ University of Michigan, Ann Arbor, MI, 48109, USA
}

\begin{abstract}
A fusion hybrid reactor whose fusion component is the gasdynamic mirror (GDM) is proposed as an energy source for a space propulsion system. The primary role of the fusion component is to supply neutrons to a blanket containing fertile material where they will breed fissile material and simultaneously burn it to produce power. Under these conditions, the fusion component need only function at or near "breakeven" condition, a much less stringent condition than that required for a pure fusion reactor. A large aspect ratio GDM is desirable from the standpoint of MHD stability, and that allows for treatment of the system as semi-infinite. With cylindrical symmetry, the performance of the system can be addressed by two, one-dimensional equations: one that describes the build-up of uranium233 in a thorium-232 blanket induced by the $14.1 \mathrm{MeV}$ neutrons emanating from a DT burning fusion component, and another that describes the transport of these high energy neutrons in the blanket. We find that for a reasonable design, such a hybrid reactor can produce hundreds of megawatts of thermal power per $\mathrm{cm}$ safely since it will operate as a subcritical system. When utilized for propulsion application, it is shown that it can generate a specific impulse of about 910 seconds at a thrust of about 0.455 mega-newtons. We assess such propulsive capability by addressing a round trip journey to Jupiter for the purpose of bringing to Earth $500 \mathrm{mT}$ of $\mathrm{He}^{3}$ which is known to exist abundantly in the atmosphere of that planet. Since approximately $1 \mathrm{mT}$ of $\mathrm{He}^{3}$ is required for a fusion reactor to produce about $2 \mathrm{GW}$-yrs of energy, one can readily see that $500 \mathrm{mT}$ represents an ample supply of fuel that can meet a significant portion of the world's energy needs. Assuming that the $\mathrm{He}^{3}$ on Jupiter has already been extracted and ready for shipment, the proposed propulsion system can make this cargo mission in about 4.7 years.
\end{abstract}

\section{Nomenclature}

$\lambda \quad$ Ion-ion collision mean free path

$R_{M}$ Plasma mirror ratio

$L \quad$ Plasma length

$n_{p} \quad$ Plasma density

$\tau \quad$ Confinement time

$v_{t h} \quad$ Ion thermal velocity

$P_{i n j}$ Injection power

$\langle\sigma v\rangle$ Maxwellian averaged reaction rate

$T$ Plasma temperature

$S \quad$ Neutron source rate

$\phi \quad$ Fast neutron flux

$N^{32}$ Thorium particle density

$N^{33}$ Uranium particle density

$\sigma_{\gamma} \quad$ Radiative capture microscopic cross section

$\sigma_{f} \quad$ Fission microscopic cross section

$k_{\text {eff }}$ Number of thermal neutrons produced per fusion neutron

$\nu \quad$ Number of neutrons per fission

*Professor Emeritus, Nuclear Engineering and Radiological Sciences, Associate Fellow AIAA. 
$\Sigma_{f} \quad$ Fusion macroscopic cross section

$\Sigma_{a b}$ Total absorption macroscopic cross section

$D \quad$ Diffusion coefficient

$B_{g} \quad$ Geometric buckling

$P_{l} \quad$ Power per unit length produced in blanket

$r_{p} \quad$ Plasma radius

$T_{0} \quad$ Inlet temperature

$T_{e x}$ Exit temperature

$\dot{m}$ Mass flow rate

$C_{p} \quad$ Specific heat

$I_{s p} \quad$ Specific impulse

$F \quad$ Thrust

$\tau_{A B}$ Travel time

\section{System Description and Analysis}

The proposed propulsion system is schematically illustrated in Fig 1 . The primary components are the fusion component, in which fusion reactions take place to produce high energy neutrons, and a surrounding blanket in which the incident neutrons breed fissile material and simultaneously burn it to produce power. Our choice for the fusion component is the GDM which is linear, cylindrically symmetric, and can operate in steady state making it especially suitable for this application. Since the primary function of the fusion component is to generate neutrons, it can operate at or near "breakeven" condition which is much less stringent than that required for a pure fusion reactor. A large aspect ratio GDM is particularly desirable since it tends to be MHD stable, and allows for treating the system as a semi-infinite cylindrical geometry. Plasma confinement in such a device is based on the condition that the ion-ion collision mean free path with respect to scattering into the loss cone, i.e., $\lambda / R_{M}$, where $\lambda$ is the collision mean free path, and $R_{M}$ is the plasma mirror ratio, is much shorter than its length, $L$. Under these conditions, the plasma behaves much like a continuum, and its dynamics is governed by the gas dynamic laws. If we employ deuteriumtritium (DT) fusion reactions in the GDM, then the $14.1 \mathrm{MeV}$ neutrons will impinge, on the average, radially onto a blanket containing thorium-232, where they will breed uranium-233 and simultaneously burn it to produce power. Using helium as a coolant flowing in ducts running along the length of the blanket, energy is extracted and converted into thrust upon exiting from a nozzle at the end of the flow. The use of the thorium fuel cycle is particularly significant since it allows for self-fueling of the reactor, while providing security since it is known to be resistant to proliferation and clandestine operations.

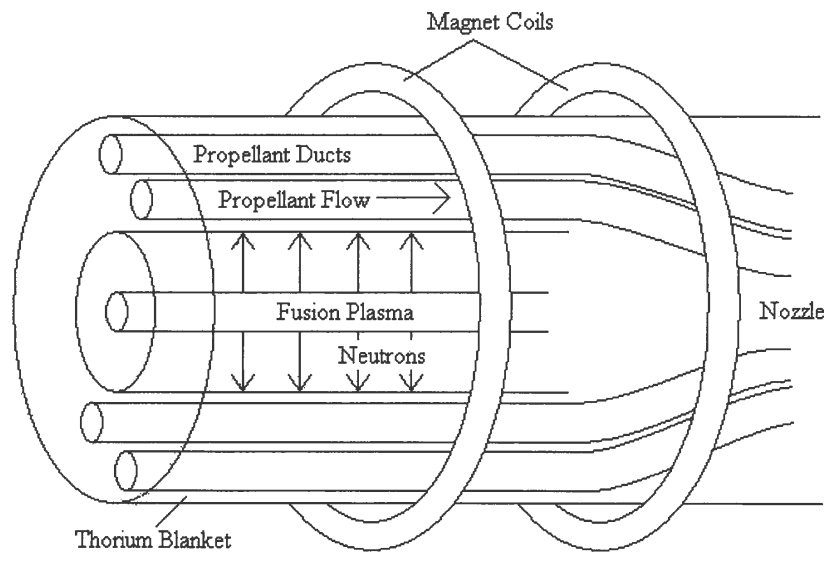

Figure 1. Fusion-fission hybrid propulsion system.

The gasdynamic condition, alluded to earlier, requires that the ion-ion collision mean free path with respect to scattering into the loss cone, i.e., $\lambda / R_{M}$ to be much less than the plasma length. For a plasma density, $n_{p}$, of $10^{16} \mathrm{~cm}^{-3}$ and temperatures of $10 \mathrm{keV}$, this condition leads to $L \geq 2.28 \mathrm{~m}^{1}{ }^{\text {Moreover, this }}$ 
length has to be reconciled with the value obtained from the confinement time, $\tau$, given by,

$$
\tau=\frac{R_{M} L}{v_{t h}}
$$

where $v_{t h}$ is the ion thermal velocity. ${ }^{2}$ For the same plasma parameters, and $R_{M}=55$, considered optimum for stability, ${ }^{3}$ Eq. (1) yields $L=183 \mathrm{~m}$ for the "breakeven" condition of DT at $10 \mathrm{keV}$, i.e.,

$$
\left(n_{p} \tau\right)_{B E}=10^{14}
$$

This length might be considered prohibitively large, and therefore we choose to operate at

$$
n_{p} \tau=5 \times 10^{-2}\left(n_{p} \tau\right)_{B E}
$$

which yields for the same plasma parameters,

$$
L=9.10 \mathrm{~m}
$$

This more reasonable length comes at a price, namely that the injection power, $P_{i n j}$ is about 10 times larger per unit length than the fusion power, assuming that the GDM is operating at a $\mathrm{Q}$ value of about unity, as predicted by the power balance in the device. For $50 \%-50 \%$ DT mixture the number of neutrons produced in the fusion component per $\mathrm{cm}^{3}$ per second is given by,

$$
n=\frac{n_{p}^{2}}{4}\langle\sigma v\rangle
$$

where $\langle\sigma v\rangle$ is the Maxwellian averaged reaction rate. This yields for $n_{p}=10^{16}$, and $T=10 \mathrm{keV}$, the value

$$
S=n=0.25 \times 10^{16} \mathrm{~cm}^{-3} \mathrm{sec}^{-1}
$$

where we denote by " $S$ " the neutron source impinging on the thorium blanket. These $14.1 \mathrm{MeV}$ neutrons breed U-233 in accordance with

$$
{ }_{90} \operatorname{Th}^{232}(n, \gamma){ }_{90} \mathrm{Th}^{233} \underset{\beta^{-}}{\stackrel{22 \mathrm{~m}}{\longrightarrow}} 91 \mathrm{~Pa}^{233} \underset{\beta^{-}}{\stackrel{24.7 \mathrm{~d}}{\longrightarrow}}{ }_{92} \mathrm{U}^{233}
$$

and, in effect, burn the U-233 to produce energy. We can establish the performance of this reactor by solving the U-233 production equation in steady state, namely,

$$
\frac{d N^{33}}{d t}=\phi \sigma_{\gamma} N^{32}-\phi \sigma_{f} N^{33}=0
$$

where $\phi$ is the fast neutron flux, $N^{32}$ and $N^{33}$ are the thorium and uranium particle densities respectively, and $\sigma_{\gamma}$ and $\sigma_{f}$ are respectively the radiative capture and fission microscopic cross sections. For determination of the power density and distribution in the blanket, one needs to calculate the neutron flux, $\phi$, from a diffusion equation that describes a "sub-critical" system where the neutron source is given by Eq. (6). Although that is readily achieveable, it may not be particularly useful, since in the presence of a moderator, the propellant (coolant) in this case, these neutrons tend to thermalize in about 10 microseconds giving rise to many thermal neutrons which in turn induce fission in U-233 to produce power. The number of thermal neutrons generated per fusion neutron can be obtained from ${ }^{4}$

$$
k_{e f f}=\frac{\nu \Sigma_{f}}{\Sigma_{a b}+D B_{g}^{2}}
$$

where $\nu(\sim 2.5)$ is the number of neutrons per fission, $\Sigma_{f}$ is the macroscopic fission cross section, $\Sigma_{a b}$ is the total (thorium plus uranium) absorption cross section, $D$ is the diffusion coefficient, and $B_{g}$ the geometric buckling. By incorporating the "poison" boron at $6 \%$ of throium density, we can generate a value of $k_{\text {eff }}$ of 0.99 which quarantees "subcriticality" and also generates,

$$
n_{t h}=\frac{1}{1-k_{e f f}}=100
$$


100 thermal neutrons per fusion neutron. This, in turn, allows us to calculate the power per unit length, $P_{l}$ produced in the blanket in accordance with,

$$
P_{l}=\pi r_{p}^{2} S n_{t h} E \Sigma_{f} / \Sigma_{t}
$$

where $r_{p}$ is the plasma radius, $S$ the neutron source given by Eq. (6), $E \approx 200 \mathrm{MeV}$, the energy produced per fission, and $\Sigma_{f} / \Sigma_{t}=0.4$, the ratio of fission to total macroscopic cross sections in the blanket. For $r_{p}=5$ $\mathrm{cm}$, Eq. (11) yields $246 \mathrm{MW} / \mathrm{cm}$ assuming that the coolant (propellant) ducts occupy $25 \%$ of the blanket cross section and $6 \mathrm{MW} / \mathrm{cm}$ of re-circulated power to the injector. From Eq. (8) we see that the number density of U-233, i.e., $N^{33}$ in steady state is given by,

$$
\frac{N^{33}}{N^{32}}=\frac{\sigma_{\gamma}}{\sigma_{f}}
$$

which gives a value of 0.1 when the cross sections are averaged over the spectral distribution of the fast neutrons. ${ }^{5}$ If we choose a blanket radius, $R=4 \mathrm{~m}$, and noting that thorium density is about $10^{4} \mathrm{~kg} / \mathrm{m}^{3}$, then for length of $9.1 \mathrm{~m}$, the mass of the blanket is about $4000 \mathrm{mT}$. The total power produced by the reactor is $224 \mathrm{GW}$. Noting also that the burn-up of U-233 at that power is $1 \mathrm{~kg} / \mathrm{d}$ per GW, then such a reactor will provide power for 1800 days assuming that the fraction of U-333 in the blanket is 1/10 that of thorium as noted above.

We turn now to assessing the propulsive capability of the proposed system by estimating the travel time it takes it to go to Jupiter to bring back to Earth $500 \mathrm{mT}$ of $\mathrm{He}^{3}$, a fuel proposed for terrestrial fusion power reactors. Using helium as the propellant, we calculate its exhaust temperature from the standard heat transfer formula:

$$
T_{e x}=T_{0}+\frac{P_{l}}{\dot{m} C_{p}} z
$$

where we assume that the inlet temperature, $T_{0}=0$ and using a mass flow rate $\dot{m}=100 \mathrm{~kg} / \mathrm{sec}$, a specific heat $C_{p}=5.2 \mathrm{~J} / \mathrm{g}-\mathrm{K}, P_{l}=246 \mathrm{MW} / \mathrm{cm}$, and $z=L=910 \mathrm{~cm}$. The exit temeprature, $T_{e x}$ is found to be $4.31 \times 10^{5} \mathrm{~K}$ or $36 \mathrm{eV}$, which translates to an exhaust velocity $v=9.1 \times 10^{3} \mathrm{~m} / \mathrm{s}$ or an $I_{s p}=910$ seconds, and a thrust $F=455$ kilonewtons. An estimate of the mass of the fusion component is $573 \mathrm{mT}$, of the blanket, $4030 \mathrm{mT}$ leading to a total mass of the vehicle of $4603 \mathrm{mT}$. If we assume the tankage mass is 230 $\mathrm{mT}$, then the dry mass of the rocket becomes $4833 \mathrm{mT}$. We employ these parameters in calculating the time it takes the propulsion device to go to Jupiter to bring back to Earth $500 \mathrm{mT}$ of $\mathrm{He}^{3}$ which is known to exist in abundance in the atmosphere of that planet. It is known that $1 \mathrm{mT} \mathrm{of} \mathrm{He}^{3}$ is required for a fusion reactor burning $\mathrm{D} \mathrm{He}^{3}$ fuel, to produce about $2 \mathrm{GW}$-years of energy, hence $500 \mathrm{mT}$ can provide fuel for many such reactors to produce power for many years. Using constant thrust, continuous burn acceleration / deceleration type of trajectory, we can write for a one-way journey to a destination " $B$ " where linear distance from a starting point " $A$ " is " $D$ ", the travel time $\tau_{A B}$ is given by, ${ }^{6}$

$$
\tau_{A B}=\frac{D}{g I_{s p}}+2 \sqrt{\frac{D m_{f}}{F}}
$$

with $m_{f}$ being the dry mass of the vehicle. In the present example, $D=6.3 \times 10^{11} \mathrm{~m}, I_{s p}=910 \mathrm{~s}$, $F=455 \times 10^{3} \mathrm{~N}, g=10 \mathrm{~m} / \mathrm{s}^{2}, m_{f}=4833 \mathrm{mT}$, the result is $\tau_{A B}=857$ days. On the return trip, the dry mass will include the payload $500 \mathrm{mT}$ so that $\tau_{B A}=860$, making the total trip time about 1717 days. We recall that this fusion-hybrid reactor will produce the required amount of power for 1800 days, thereby allowing the system sufficient time to possibly extract the needed amount of $\mathrm{He}^{3}$ while circling the planet. It may be interesting to compare the mission to Jupiter to bring to earth $500 \mathrm{mT}$ of $\mathrm{He}^{3}$, with that to the atmospheres of Uranus and Neptune for the same purpose. ${ }^{7}$ The Uranus mission required 23 years for the round trip. It required, using nuclear thermal propulsion, 4 years to arrive at Uranus, 11 years for the mining operation, and 8 years to return to Earth. In the Jupiter mission, it was assumed that the He-3 had somehow been previously extracted or can be extracted and loaded in 83 days so that the 1800 days of self-fueling can be utilized to accomplish the mission. 


\section{Conclusion}

We have introduced a propulsion system based on the fusion hybrid reactor, in which energy is produced by fission reactions in a thorium blanket induced by fusion generated neutrons. Uranium-233 was bred in the blanket and simultaneously burned to produce power. As a result, the propulsion system is self-fueling, and with the design presented, it is shown that it can make a journey to Jupiter to bring back to Earth 500 $\mathrm{mT}$ of $\mathrm{He}^{3}$ in approximately 4.7 years.

\section{Acknowledgments}

The author is grateful to B. Weatherford and I. Bell for their help in the preparation of this manuscript.

\section{References}

\footnotetext{
${ }^{1}$ Kammash, T., "A Fusion Hybrid Reactor Based on the Gasdynamic Mirror (GDM)," Trans. ICENES 2011 Conference, San Francisco, CA, May 15-19 (2011)

${ }^{2}$ Kammash, T. and Lee, M.J., J. Propulsion and Power, Vol. 11, 544 (1995)

${ }^{3}$ Post, R.F. and Ryutov, D.D., Trans. of Fusion Technology, Vol. 27, 117 (1995)

${ }^{4}$ Duderstadt, J.J. and Hamilton, L.J., Nuclear Reactor Analysis, John Wiley \& Sons, Inc. (1975)

${ }^{5}$ Rubia, C., AIP Conference Proceedings, Las Vegas, NV, Vol. 346 (1994)

${ }^{6}$ Kammash, T., Fusion Energy in Space Propulsion, AIAA Progress in Astronautics and Aeronautics, Vol. 167 (1995),

${ }^{7}$ Palaszewski, B., AIAA/ASME/SAE/ASEE Joint Propulsion Conference, Paper AIAA-2010-6573 (2010)
} Chapter 1 